\title{
Predictions for the Neutrino Parameters in the Minimal Model Extended by General Lepton Flavor-Dependent U(1) Gauge Symmetries
}

\author{
Kento Asai \\ Department of Physics, University of Tokyo, Bunkyo-ku, Tokyo 133-0033, Japan
}

\begin{abstract}
Lepton flavor-dependent U(1) gauge symmetries give strong constraints on the neutrino Dirac and Majorana mass matrices, and, in the cases of some kinds of $U(1) s$, the mass matrix for the light neutrinos have some zero elements. We study the minimal extensions of the Standard Model by a linear combination of $\mathrm{U}(1)_{L_{e}-L_{\mu}}, \mathrm{U}(1)_{L_{\mu}-L_{\tau}}$ and $\mathrm{U}(1)_{B-L}$ gauge symmetries, which realizes the two-zero minor or texture structure in the mass matrix for the active neutrinos. Analyzing these structures of the neutrino mass matrix, we obtain the predictions for the neutrino parameters, such as the neutrino masses and Dirac CP phase. In addition, we also discuss the implication of our results for leptogenesis.
\end{abstract}

Keywords: Flavor symmetry, Neutrino Physics

DOI: $10.31526 /$ ACP.NDM-2020.10

\section{INTRODUCTION}

Lepton flavor-dependent U(1) gauge symmetries are one of the possibilities of extensions of the gauge sector in the Standard Model. It is well known that $\mathrm{U}(1)_{L_{\alpha}}-L_{\beta}$, where $L_{\alpha}$ is the lepton number of generation associated with $\alpha(=e, \mu, \tau)$, can be introduced to the gauge group of the Standard Model (SM). Especially, $\mathrm{U}(1)_{L_{\mu}-L_{\tau}}$ gauge symmetry is often discussed in the context of the muon anomalous magnetic moment $[1,2,3,4,5,6]$. In models extended by some lepton flavor-dependent U(1) gauge symmetries, mass matrix for the active neutrinos or its inverse matrix has two-zero elements, and these kind of structures are called two-zero texture (TZT) and two-zero minor(TZM) structures, respectively. In Refs. [7, 8], the relations between the two-zero minor and texture structures and neutrino parameters have been analyzed, and the predictions for the neutrino masses, Dirac CP phase, and so on, have been obtain as functions of the neutrino oscillation parameters. As shown in Ref. [8], the minimal gauged $U(1)_{L_{u}}-L_{\tau}$ is facing severely constraints from the Planck 2018 limit [21] on the sum of the neutrino masses, and so we have extended U(1) $L_{L_{\mu}}-L_{\tau}$ gauge symmetries to linear combinations of $U(1)_{L_{e}-L_{\mu}}, \mathrm{U}(1)_{L_{\mu}-L_{\tau}}$ and $\mathrm{U}(1)_{B-L}$ gauge symmetries [9].

Here we briefly report the analyses of the minimal models extended by general lepton flavor-dependent $U(1)$ gauge symmetries, following Ref. [9].

\section{MINIMAL GAUGED U(1) MODELS AND NEUTRINO MASS MATRICES}

Here, we consider the following extra $U(1)_{Y^{\prime}}$ gauge symmetry:

- All quarks have the same $\mathrm{U}(1)_{Y^{\prime}}$ charges.

- Left-handed and right-handed fermions have the same $\mathrm{U}(1)_{Y^{\prime}}$ charges.

- Charged leptons and neutrinos with the same flavor have the same $U(1)_{Y^{\prime}}$ charge.

- $\mathrm{U}(1)_{Y^{\prime}}$ charge of the Higgs field is zero.

In order to satisfy the condition of anomaly cancellation, the $U(1)_{Y^{\prime}}$ charge should be written as follows $[10,11,12]$ :

$$
Y^{\prime}= \begin{cases}x_{e} L_{e}+x_{\mu} L_{\mu}-\left(x_{e}+x_{\mu}\right) L_{\tau} & \text { (B independent) } \\ B+x_{e} L_{e}+x_{\mu} L_{\mu}-\left(3+x_{e}+x_{\mu}\right) L_{\tau} & \text { (B dependent) }\end{cases}
$$

where $B$ is the baryon number, and $x_{\alpha}(\alpha=e, \mu, \tau)$ is the free real number. Different combinations of the coefficients $\left(x_{\alpha}\right)$ realize different $\mathrm{U}(1)$ gauge symmetries, and among them, $15 \mathrm{U}(1)_{Y^{\prime}} \mathrm{s}$ realize the TZM structures and $3 \mathrm{U}(1)_{Y^{\prime}} \mathrm{S}$ realize the TZT structures within the framework of minimal gauged $\mathrm{U}(1)$ models. Here, we show the case of $\mathrm{U}(1)_{B+L_{e}-3 L_{\mu}-L_{\tau}}$ with a $\mathrm{SM}$ singlet $\mathrm{U}(1)_{Y^{\prime}}-$ breaking scalar as example. 
The interaction terms relevant to the lepton masses are given by

$$
\begin{aligned}
\Delta \mathcal{L}= & -y_{e} e_{R}^{c} L_{e} H^{\dagger}-y_{\mu} \mu_{R}^{c} L_{\mu} H^{\dagger}-y_{\tau} \tau_{R}^{c} L_{\tau} H^{\dagger} \\
& -\lambda_{e} N_{e}^{c}\left(L_{e} \cdot H\right)-\lambda_{\mu} N_{\mu}^{c}\left(L_{\mu} \cdot H\right)-\lambda_{\tau} N_{\tau}^{c}\left(L_{\tau} \cdot H\right) \\
& -M_{e \tau} N_{e}^{c} N_{\tau}^{c}-\frac{1}{2} \lambda_{e e} \sigma N_{e}^{c} N_{e}^{c}-\lambda_{e \mu} \sigma^{*} N_{e}^{c} N_{\mu}^{c}-\frac{1}{2} \lambda_{\tau \tau} \sigma^{*} N_{\tau}^{c} N_{\tau}^{c} \\
& + \text { h.c. }
\end{aligned}
$$

where the dots indicate the contraction of the $\mathrm{SU}(2)_{L}$ indices. After the Higgs field $H$ and $\mathrm{U}(1)_{Y^{\prime}}$-breaking scalar $\sigma$ acquire the vacuum expectation values (VEVs), the charged lepton, neutrino Dirac, and Majorana mass matrices are given by

$$
\begin{aligned}
\mathcal{M}_{\ell} & =\frac{v}{\sqrt{2}}\left(\begin{array}{ccc}
y_{e} & 0 & 0 \\
0 & y_{\mu} & 0 \\
0 & 0 & y_{\tau}
\end{array}\right), \mathcal{M}_{D}=\frac{v}{\sqrt{2}}\left(\begin{array}{ccc}
\lambda_{e} & 0 & 0 \\
0 & \lambda_{\mu} & 0 \\
0 & 0 & \lambda_{\tau}
\end{array}\right), \\
\mathcal{M}_{R} & =\left(\begin{array}{ccc}
\lambda_{e e}\langle\sigma\rangle & \lambda_{e \mu}\langle\sigma\rangle & M_{e \tau} \\
\lambda_{e \mu}\langle\sigma\rangle & 0 & 0 \\
M_{e \tau} & 0 & \lambda_{\tau \tau}\langle\sigma\rangle
\end{array}\right) .
\end{aligned}
$$

As shown in Eq. (3), the charged lepton and neutrino Dirac mass matrices are diagonal, and the $(\mu, \mu)$ and $(\mu, \tau)$ elements in the Majorana mass matrices vanish. Here, we assume that the Majorana masses are much heavier than the Dirac masses, and then by the seesaw mechanism $[13,14,15,16]$, the mass matrix for the active neutrinos is given by

$$
\mathcal{M}_{v_{L}} \simeq-\mathcal{M}_{D} \mathcal{M}_{R}^{-1} \mathcal{M}_{D}^{T}
$$

Because of the matrix structure of the Majorana mass, he inverse matrix of $\mathcal{M}_{v_{L}}$ also has zero elements in the $(\mu, \mu)$ and $(\mu, \tau)$

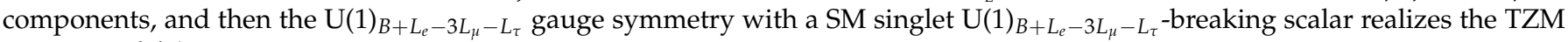
structure of $\mathcal{M}_{v_{L}}$.

\section{VANISHING CONDITIONS AND NEUTRINO PARAMETERS}

Let us show the analyses of the TZM structures, following Refs. [9]. The mass eigenvalues of the active neutrinos are obtained by diagonalizing $\mathcal{M}_{v_{L}}$ like

$$
U_{\mathrm{PMNS}}^{T} \mathcal{M}_{v_{L}} U_{\mathrm{PMNS}}=\operatorname{diag}\left(m_{1}, m_{2}, m_{3}\right),
$$

where $U_{\text {PMNS }}$ is the so-called Pontecorvo-Maki-Nakagawa-Sakata (PMNS) mixing matrix $[17,18,19,20]$. Combining Eq. (4) and (5), we obtain the following equation:

$$
\begin{aligned}
\mathcal{M}_{v_{L}}^{-1} & =-\left(\mathcal{M}_{D}^{-1}\right)^{T} \mathcal{M}_{R} \mathcal{M}_{D}^{-1} \\
& =U_{\text {PMNS }} \operatorname{diag}\left(m_{1}^{-1}, m_{2}^{-1}, m_{3}^{-1}\right) U_{\text {PMNS }}^{T}
\end{aligned}
$$

where $m_{i}$ is the mass eigenvalues of the active neutrinos. As mentioned above, the mass matrix for the active neutrinos has the TZM structure and its $(\mu, \mu)$ and $(\mu, \tau)$ elements vanish, and then those components in the right-hand side should also vanish. These conditions, hereafter written as vanishing conditions, are concretely written as follows:

$$
\begin{aligned}
\frac{1}{m_{1}} V_{\mu 1}^{2}+\frac{1}{m_{2}} V_{\mu 2}^{2} e^{i \alpha_{2}}+\frac{1}{m_{3}} V_{\mu 3}^{2} e^{i \alpha_{3}} & =0, \\
\frac{1}{m_{1}} V_{\mu 1} V_{\tau 1}+\frac{1}{m_{2}} V_{\mu 2} V_{\tau 2} e^{i \alpha_{2}}+\frac{1}{m_{3}} V_{\mu 3} V_{\tau 3} e^{i \alpha_{3}} & =0,
\end{aligned}
$$

where the unitary matrix $V$ is defined by $U_{\mathrm{PMNS}}=V \cdot \operatorname{diag}\left(1, e^{i \alpha_{2} / 2}, e^{i \alpha_{3} / 2}\right)$ and $\alpha_{2}, \alpha_{3}$ are the Majorana CP phases. By solving two complex equations, namely the vanishing conditions in Eq. (7) and (8), the lightest mass eigenvalue of the active neutrinos $m_{1}$, Dirac CP phase $\delta$, and Majorana CP phases $\alpha_{2,3}$ can be obtained as functions of the neutrino oscillation parameters, such as the mixing angles $\theta_{12}, \theta_{23}, \theta_{13}$ and mass squared differences $\Delta m_{21}^{2}, \Delta m_{32}^{2}$. As shown in Eq. (7) and (8), the vanishing conditions do not depend on the VEV of the $\mathrm{U}(1)_{B+L_{e}-3 L_{\mu}-L_{\tau}}$-breaking scalar $\langle\sigma\rangle$ and Majorana masses $M_{\mathcal{e} \tau}$, and then the obtained neutrino parameters are also independent of the Majorana mass scale.

\section{PREDICTIONS FOR THE NEUTRINO PARAMETERS}

Here let us show the results of analyses of the vanishing conditions. Among the $15 \mathrm{U}(1)_{Y^{\prime}} \mathrm{s}$ which realize the TZM structures and 3 $\mathrm{U}(1)_{Y^{\prime}} \mathrm{S}$ which realize the TZT structures, only $3 \mathrm{U}(1)_{Y^{\prime}} \mathrm{S}$ which realize the TZM structures have physical solutions and are consistent 
TABLE 1: Lepton flavor-dependent U(1) gauge symmetries and structural patterns of the two-zero minor structures.

\begin{tabular}{|c||ccc|}
\hline$Y^{\prime}$ & $L_{\mu}-L_{\tau}$ & $B+L_{e}-3 L_{\mu}-L_{\tau}$ & $B+L_{e}-L_{\mu}-3 L_{\tau}$ \\
\hline \multirow{M}{\mathcal{M}_{v_{L}}^{-1}}{} & $\left(\begin{array}{ccc}* & * & * \\
* & 0 & * \\
* & * & 0\end{array}\right)$ & $\left(\begin{array}{ccc}* & * & * \\
* & 0 & 0 \\
* & 0 & *\end{array}\right)$ & $\left(\begin{array}{ccc}* & * & * \\
* & * & 0 \\
* & 0 & 0\end{array}\right)$ \\
\hline
\end{tabular}

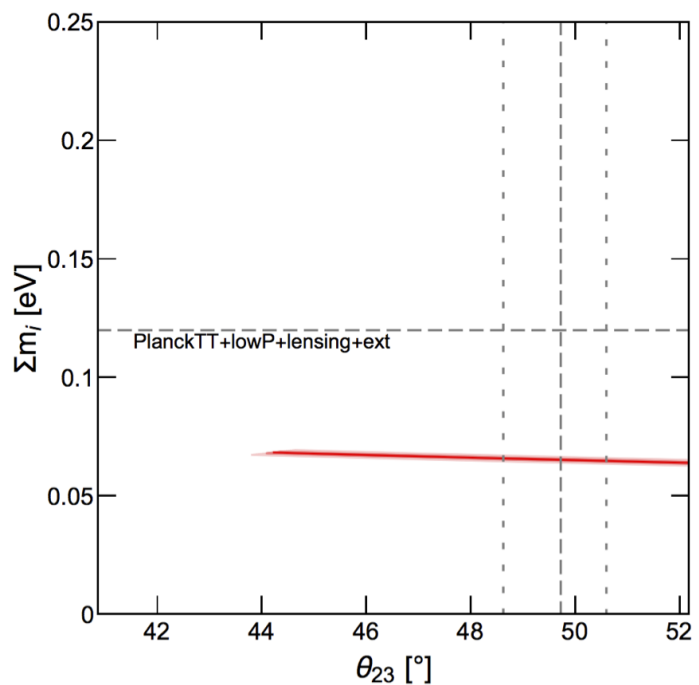

FIGURE 1: The prediction for the sum of the neutrino masses in $U(1)_{B+L_{e}-3 L_{\mu}-L_{\tau}}$ (NO) case.

with the Planck 2018 limit on the sum of the neutrino masses, and in all cases, the normal mass ordering of the neutrino masses is favored. In Tab. 1, we show these $3 \mathrm{U}(1)_{Y^{\prime}} \mathrm{s}$ and mass structures. In Refs. [8], the $\mathrm{U}(1)_{L_{\mu}-L_{\tau}}$ case have been discussed in detail, and we show the results of the $\mathrm{U}(1)_{B+L_{e}-3 L_{\mu}-L_{\tau}}$ case.

In Fig. 1, we show the prediction for the sum of the neutrino masses. The red line shows the prediction as functions of $\theta_{23}$ and the dark (light) red bands show the uncertainty coming from the $1 \sigma(3 \sigma)$ errors of $\theta_{13}$ and $\Delta m^{2}$. The vertical gray dashed line represents the best fit value of $\theta_{23}$ and the vertical gray dotted lines represent the $1 \sigma$ region. The horizontal dashed gray line shows the present limit on the sum of the neutrino masses by the Planck experiment: $\sum_{i} m_{i}<0.12 \mathrm{eV}$ (Planck TT+lowP+lensing+ext) [21]. As shown in Fig. 1, the predicted value is below the Planck 2018 limit.

\section{IMPLICATIONS FOR LEPTOGENESIS}

The minimal gauged $\mathrm{U}(1)_{Y^{\prime}}$ models contain 3 right-handed neutrinos, and then if their masses are large enough, the baryon asymmetry of the Universe can be generated through the leptogenesis scenario [22]. In the leptogenesis scenario, the lepton number is generated by decay of heavy neutrinos, and then the lepton number are converted into the baryon number through the sphaleron process [23]. Between the baryon $n_{B}$ and lepton densities $n_{L}$ generated through the leptogenesis scenario, $n_{B} / n_{L}<0$ satisfies, and the observed baryon number is positive. Therefore, the lepton number generated by the decay of the heavy neutrinos should be negative.

As we discussed in the previous section, because of the vanishing conditions, the neutrino parameters in the minimal gauged $\mathrm{U}(1)_{Y^{\prime}}$ models are obtained as functions of the neutrino oscillation parameters. Therefore, from Eq. (6), the neutrino Majorana masses can be written as functions the neutrino oscillation parameters and three free parameters, namely the Dirac Yukawa couplings. Now, we parametrize the neutrino Dirac Yukawa couplings as follows:

$$
\left(\lambda_{e}, \lambda_{\mu}, \lambda_{\tau}\right)=\lambda(\cos \theta, \sin \theta \cos \phi, \sin \theta \sin \phi)
$$

Using the predictions for the three CP phases and lightest neutrino mass in the previous section, we calculate the sign of the lepton number generated the decay of the heavy neutrinos, and we show the result in Fig. 2 . The red shaded areas show the parameter region of the $(\theta, \phi)$ plane where the lepton number generated by the decay of the heavy neutrinos is negative. Moreover, we also show the contours of the right-handed neutrino mass ratio $M_{2} / M_{1}$. As shown Fig. 2, in almost all parameter space, the correct sign of the lepton number is realized in $\mathrm{U}(1)_{B+L_{e}}-3 L_{\mu}-L_{\tau}$ (NO) case. 


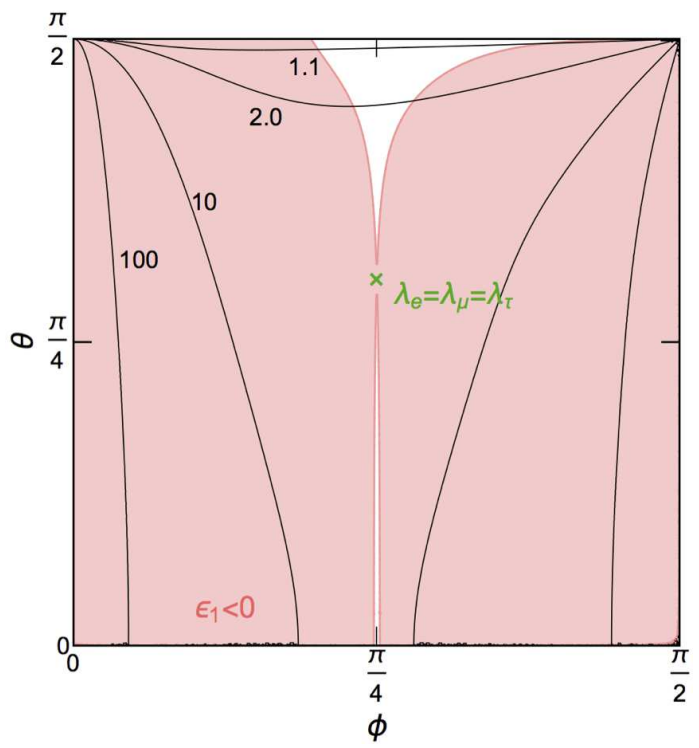

FIGURE 2: The predictions for the sign of the lepton number in $\mathrm{U}(1)_{B+L_{e}-3 L_{u}-L_{\tau}}(\mathrm{NO})$ case.

\section{SUMMARY}

We have examined the models extended by a linear combination of $U(1)_{L_{e}-L_{\mu}}, \mathrm{U}(1)_{L_{\mu}-L_{\tau}}$ and $\mathrm{U}(1)_{B-L}$ gauge symmetries, which realizes the two-zero minor or texture structure in the mass matrix for the active neutrinos. Analyzing the vanishing conditions, we have obtained the predictions for the neutrino $\mathrm{CP}$ phases and masses as functions of the neutrino oscillation parameters, and have found that only $3 \mathrm{U}(1)_{Y^{\prime}} \mathrm{s}$ with a $Y^{\prime}$-breaking singlet scalar cases are consistent with the recent experiments and all case favor the normal ordering. Moreover, we have discussed the implications for leptogenesis. Using the predictions for the three CP phases and lightest neutrino mass, we have calculated the sign of the lepton number which is generated by the decay of the heavy neutrinos, and have discussed whether the correct sign of the baryon asymmetry of the Universe can be realize or not.

\section{ACKNOWLEDGEMENTS}

This work was supported by JSPS KAKENHI Grant Number 19J13812.

\section{References}

[1] G. W. Bennett et al. [Muon g-2 Collaboration], Phys. Rev. D 73, 072003 (2006) doi:10.1103/PhysRevD.73.072003 [hep-ex/0602035].

[2] F. Jegerlehner and A. Nyffeler, Phys. Rept. 477, 1 (2009) doi:10.1016/j.physrep.2009.04.003 [arXiv:0902.3360 [hep-ph]].

[3] M. Davier, A. Hoecker, B. Malaescu and Z. Zhang, Eur. Phys. J. C 71, 1515 (2011) Erratum: [Eur. Phys. J. C 72, 1874 (2012)] doi:10.1140/epjc/s10052-012-1874-8, 10.1140/epjc/s10052-010-1515-z [arXiv:1010.4180 [hep-ph]].

[4] K. Hagiwara, R. Liao, A. D. Martin, D. Nomura and T. Teubner, J. Phys. G 38, 085003 (2011) doi:10.1088/0954-3899/38/8/085003 [arXiv:1105.3149 [hep-ph]].

[5] S. Baek, N. G. Deshpande, X. G. He and P. Ko, Phys. Rev. D 64, 055006 (2001) doi:10.1103/PhysRevD.64.055006 [hep-ph/0104141].

[6] E. Ma, D. P. Roy and S. Roy, Phys. Lett. B 525, 101 (2002) doi:10.1016/S0370-2693(01)01428-9 [hep-ph/0110146].

[7] K. Asai, K. Hamaguchi and N. Nagata, Eur. Phys. J. C 77, no. 11, 763 (2017) doi:10.1140/epjc/s10052-017-5348-x [arXiv:1705.00419 [hep-ph]].

[8] K. Asai, K. Hamaguchi, N. Nagata, S. Y. Tseng and K. Tsumura, Phys. Rev. D 99, no. 5, 055029 (2019) doi:10.1103/PhysRevD.99.055029 [arXiv:1811.07571 [hep-ph]].

[9] K. Asai, Eur. Phys. J. C 80, no. 2, 76 (2020) doi:10.1140/epjc/s10052-020-7622-6 [arXiv:1907.04042 [hep-ph]].

[10] T. Araki, J. Heeck and J. Kubo, JHEP 1207, 083 (2012) doi:10.1007/JHEP07(2012)083 [arXiv:1203.4951 [hep-ph]].

[11] J. Heeck,

[12] C. Kownacki, E. Ma, N. Pollard and M. Zakeri, Phys. Lett. B 766, 149 (2017) doi:10.1016/j.physletb.2017.01.013 [arXiv:1611.05017 [hep-ph]].

[13] P. Minkowski, Phys. Lett. 67B, 421 (1977). doi:10.1016/0370-2693(77)90435-X

[14] T. Yanagida, Conf. Proc. C 7902131, 95 (1979).

[15] M. Gell-Mann, P. Ramond and R. Slansky, Conf. Proc. C 790927, 315 (1979) [arXiv:1306.4669 [hep-th]].

[16] R. N. Mohapatra and G. Senjanovic, Phys. Rev. Lett. 44, 912 (1980). doi:10.1103/PhysRevLett.44.912

[17] B. Pontecorvo, Sov. Phys. JETP 26, 984 (1968) [Zh. Eksp. Teor. Fiz. 53, 1717 (1967)].

[18] B. Pontecorvo, Sov. Phys. JETP 6, 429 (1957) [Zh. Eksp. Teor. Fiz. 33, 549 (1957)].

[19] B. Pontecorvo, Sov. Phys. JETP 7, 172 (1958) [Zh. Eksp. Teor. Fiz. 34, 247 (1957)].

[20] Z. Maki, M. Nakagawa and S. Sakata, Prog. Theor. Phys. 28, 870 (1962). doi:10.1143/PTP.28.870

[21] N. Aghanim et al. [Planck Collaboration], arXiv:1807.06209 [astro-ph.CO].

[22] M. Fukugita and T. Yanagida, Phys. Lett. B 174, 45 (1986). doi:10.1016/0370-2693(86)91126-3

[23] J. A. Harvey and M. S. Turner, Phys. Rev. D 42, 3344 (1990). doi:10.1103/PhysRevD.42.3344 